\title{
El aprendizaje significativo de la Física para estudiantes-atletas de las escuelas deportivas
}

Tomasa Magaly Longa-Martínez

Magíster en Fundamentos de las Ciencias de la Educación, Instituto Superior Pedagógico Enrique José Varona. Profesora asistente, Universidad de las Ciencias de la Cultura Física y el Deporte "Manuel Fajardo".

Correo electrónico:

magalylm@inder.cu, magalylm@uccfd.cu

Recibido: 1 de febrero del 2015

Aprobado: 17 de mayo del 2015

Cómo citar este artículo: Longa-Martínez, Tomasa Magaly. "El aprendizaje significativo de la Física para estudiantes-atletas de las escuelas deportivas". Rastros Rostros 17.31 (2015): 117-125. Impreso. doi: http://dx.doi. org/10.16925/ra.v17i31.1093

\section{Resumen}

Introducción: el propósito del presente artículo es explicar la importancia de lograr un aprendizaje significativo de la Física en estudiantes-atletas. Se exponen los rasgos característicos de la enseñanza desarrolladora, haciendo hincapié en el aprendizaje significativo, uno de sus pilares. Metodología: en la investigación se emplearon diferentes métodos teóricos y empíricos. Entre los primeros, se emplearon el método histórico-lógico, el análisis y la síntesis, así como el análisis de documentos. El método sistémico-estructural fue utilizado a fin de elaborar un sistema de recomendaciones metodológicas. Resultados: finalmente, se muestran los resultados y se destaca la aplicación de la estrategia con el propósito de lograr que los contenidos de la Física sean significativos para los estudiantes atletas, y así mejorar sus resultados académicos. Conclusiones: es esencial que se relacionen los contenidos y las leyes de la Física con situaciones deportivas concretas y, de esta manera, se demuestre cómo la aplicación correcta en estas regularidades contribuye al éxito deportivo.

Palabras clave: aprendizaje significativo, atletas, Física, proceso de enseñanza-aprendizaje. 


\title{
Meaningful Learning of Physics for Student Athletes at Sports Schools
}

\begin{abstract}
Introduction: the purpose of this article is to explain the importance of achieving meaningful learning of physics in student athletes. The distinctive features of developmental teaching are discussed, emphasizing meaningful learning as one of its pillars. Methodology: this research employed different theoretical and empirical methods. Among the former, the historical-logical method, analysis and synthesis, and document analysis were used. The systemic-structural method was used to develop a system of methodological recommendations. Results: finally, the results are shown and the implementation of the strategy is highlighted in order to ensure that the contents of physics are meaningful to student athletes and thus improve their academic performance. Conclusions: it is essential that the contents and laws of physics are related to specific sports situations and, thus, it is demonstrated how the correct application to these regularities contributes to athletic success.
\end{abstract}

Keywords: meaningful learning, athletes, physics, teaching-learning process.

\section{A aprendizagem significativa da Física para estudantes- atletas das escolas esportivas}

\section{Resumo}

Introdução: o escopo deste artigo é explicar a importância de alcançar um aprendizado significativo da Física em estudantes-atletas. Expõem-se os rasgos característicos do ensino desenvolvedor, enfatizando o aprendizado significativo, um dos seus pilares. Metodologia: diversos métodos teóricos e empíricos foram empregados na investigação. Entre os primeiros, empregaram-se o método histórico-lógico, a análise e a síntese, bem como a análise de documentos. $\mathrm{O}$ método sistémico-estrutural foi utilizado para construir um sistema de recomendações metodológicas. Resultados: finalmente, mostram-se os resultados e é de salientar a aplicação da estratégia para conseguir que os conteúdos da Física sejam significativos para os estudantes atletas, e dessa forma melhorar seus resultados académicos. Conclusões: é essencial que conteúdos e leis da Física sejam relacionados com situações esportivas especificas e, assim, seja demonstrado que a aplicação certa nestas regularidades contribui ao sucesso.

Palavras-chave: aprendizado significativo, atletas, Física, processo de ensino-aprendizado. 


\section{Introducción}

\section{El proceso de enseñanza-aprendizaje: características y retos}

Una de las necesidades más importantes a satisfacer por cualquier sociedad es la preparación de los ciudadanos del país, los cuales deben poseer un nivel cultural adecuado para el desarrollo de una labor eficiente. Ello se traduce en comprender la cultura como un conjunto heterogéneo de elementos diversos, tales como lo científico, lo político, lo moral, lo ideológico, lo filosófico, lo estético y lo artístico, entre otros. De este modo, el hombre podrá comprender el mundo en sus múltiples aristas y transformarlo. Es un objetivo de la sociedad que todos sus miembros sean aptos para desempeñar determinado papel entre las múltiples funciones que se llevan a cabo en ella. La Unesco declaró:

[...] que la cultura da al hombre la capacidad de reflexionar sobre sí mismo. Es ella la que hace de nosotros seres específicamente humanos, racionales, críticos y éticamente comprometidos. A través de ella el hombre se expresa, toma conciencia de sí mismo, se reconoce como un proyecto inacabado, pone en cuestión sus propias realizaciones, busca incansablemente nuevas significaciones, y crea obras que lo transcienden. (Unesco 1)

Por ello, el concepto de preparación integral es de gran importancia para la ciencia pedagógica. Así lo plantean Chávez y Deler:

Cuba es heredera de una rica tradición pedagógica, que se puede enmarcar desde la segunda mitad del siglo XVIII, en que aparece la cubaría y, este ideario educativo se fortalece a lo largo del siglo xIx y del xx, y por supuesto, en lo que va del siglo xxI, con figuras cimeras que han creado un pensamiento original, sin dejar de tener vínculos con el universal y el latinoamericano. (1)

La pedagogía cubana actual se nutre de las tradiciones pedagógicas del país y de lo más avanzado del pensamiento universal. Contempla en la escuela la instrucción que se encarga del proceso de enseñanza-aprendizaje, así como considera que es el aprendizaje la actividad que desarrolla el estudiante en su formación para aprender y asimilar la materia de estudio, mientras que el profesor es el guía del aprendizaje, quien ejecuta la enseñanza. Profesor y alumno actúan sobre la materia de estudio.

La Dra. Ana María González Fernández define la investigación educativa como:

[...] la actividad de producción científica en el campo de las Ciencias de la Educación, entendidas como sistema, que estudian la realidad educativa desde la totalidad y se aproximan en la misma medida a sus diferentes dimensiones, facetas, relaciones y procesos particulares, integrando los aportes de las Ciencias Pedagógicas, la Psicología de la Educación, la Sociología de la Educación, la Filosofía de la Educación, la Historia de la Educación, la Informática Educacional, entre otras, para construir un cuadro vivo y dinámico de este complejo fenómeno. (14)

En otras palabras, para estudiar el proceso de enseñanza-aprendizaje, es necesario emplear un método científico, lo cual conduce a un enfoque de sistema. En primer lugar, se deben analizar sus componentes $y$, luego, las relaciones que entre ellos se establecen. El proceso de enseñanza-aprendizaje es un sistema, es decir, un conjunto de elementos interrelacionados entre sí que constituyen una determinada formación integral.

La institución escolar recoge en el diseño curricular un modelo pedagógico, y este se traduce particularmente en los planes de estudio, los programas de las asignaturas o disciplinas. Por tanto, este modelo pedagógico constituye el objeto que dará una respuesta pedagógica al encargo social que ha recibido la escuela.

Dentro del sistema educativo cubano, una especificidad la constituyen las escuelas deportivas. Estas se caracterizan por integrar la formación cultural general a la deportiva, en función de formar un atleta integral (Pérez 3). Las escuelas deportivas cubanas son instituciones formadoras de atletas de alto rendimiento, y van desde las edades tempranas hasta la edad juvenil. Se caracterizan por tener una base material deportiva y un currículo deportivo, cuyo núcleo es el "Plan de Preparación Deportiva", y un currículo de formación general, el cual se utiliza en las escuelas del Ministerio de Educación para todos los estudiantes (este último se adecúa en el tiempo y en las actividades a las condiciones del atleta). Las escuelas deportivas están organizadas de modo que los estudiantes-atletas puedan entrenar y cumplir con los calendarios de competencia y reciban la atención 
necesaria, a fin de que puedan vencer las asignaturas de la enseñanza general.

La escuelas deportivas deben propiciar en los estudiantes-atletas un proyecto de vida más allá de su accionar deportivo presente. Al terminar la enseñanza media, muchos de ellos ingresan en la Licenciatura en Cultura Física; otros, a carreras universitarias de diverso perfil.

Con relación a la asignatura "Física", es imprescindible que los atletas-estudiantes comprendan cómo su dominio puede contribuir a un mejor desempeño deportivo. La reciben, como el resto de los estudiantes de la enseñanza general, en los tres grados del preuniversitario.

El mejoramiento en la educación requiere transformaciones más allá de los programas docentes. La elevación de la calidad del proceso de enseñanza-aprendizaje en general, y el de las escuelas deportivas, transita por tales cambios. Entre las opciones que se están aplicando para alcanzar ese objetivo, está la educación desarrolladora.

Se pueden destacar las siguientes ideas claves presentes en las concepciones pedagógicas de la educación desarrolladora (Castellanos 15):

- La intencionalidad, al considerar definitorio el planteamiento de determinados propósitos y fines conscientes, que se objetivan e instrumentan en la modelación del cambio: el logro de una educación desarrolladora es la finalidad última del cambio, lo cual permite establecer su direccionalidad (el para qué).

- Las concepciones, actitudes y prácticas de la comunidad educativa escolar como objeto del cambio (el qué).

- La contextualización del modelo en correspondencia con los escenarios socioeconómicos actuales y futuros, considerando en cada momento la dialéctica de lo posible y lo real, así como la articulación de las demandas presentes con la visión de desarrollo futuro.

- Entre los problemas que se han apreciado en algunas escuelas del país, se encuentra el que los contenidos curriculares en ocasiones carecen de pertinencia y significatividad, tanto individual como social, y se ofrecen a partir de la lógica de las disciplinas independientes de forma aislada y desconectada, situación que se agudiza en la secundaria básica. Los estudios fueron realizados por especialistas del Instituto Superior Pedagógico Enrique José Varona.
El problema de la significatividad es uno de los aspectos esenciales planteados. Muchos estudiantes llegan a la educación preuniversitaria sin un aprendizaje significativo y se afecta la calidad del proceso docente educativo en ese nivel.

Es oportuno recordar que la educación cubana, además de partir de las tradiciones del país - a lo largo de la historia los principales intelectuales han sido también maestros-, toma como base también otras concepciones, entre las cuales se halla el paradigma histórico-cultural de Vigotsky.

En el trabajo Hacia un aprendizaje desarrollador, de un colectivo de autores del Centro de Estudios Educacionales del Instituto Superior Pedagógico (ISP) Enrique José Varona, se establecen algunas conclusiones esenciales acerca de la relación dialéctica existente entre la educación, el aprendizaje y el desarrollo en el ser humano:

- Educación, aprendizaje y desarrollo son procesos que poseen una relativa independencia y singularidad propia, pero que se integran al mismo tiempo en la vida humana, conformando una unidad dialéctica.

- Se entiende que una educación desarrolladora es aquella que conduce al desarrollo, que va delante - guiando, orientando, estimulando-, que tiene en cuenta el desarrollo actual para ampliar continuamente los límites de la zona de desarrollo próximo o potencial, y por lo tanto, los progresivos niveles de desarrollo del sujeto. La educación desarrolladora promueve y potencia los aprendizajes desarrolladores (Castellanos 23).

Además, para estos autores el aprendizaje desarrollador está conformado por tres dimensiones: significatividad, motivación para aprender y carácter activo. Sobre el aprendizaje significativo - que consideran muy importante- versa el contenido del presente artículo. Además, plantean otros aspectos importantes acerca del aprendizaje:

- Aprender es un proceso que ocurre a lo largo de toda la vida, y que se extiende en múltiples espacios, tiempos y formas. El aprender está estrechamente ligado con el crecer de manera permanente. Sin embargo, no es algo abstracto: está vinculado a las experiencias vitales y las necesidades de los individuos, a su contexto histórico-cultural concreto.

- En el aprendizaje cristaliza continuamente la dialéctica entre lo histórico-social y lo individual-personal; es siempre un proceso activo de 
reconstrucción de la cultura y de descubrimiento del sentido personal y la significación vital que tiene el conocimiento para los sujetos.

- Aprender supone el tránsito de lo externo a lo interno - en palabras de Vigotsky, de lo interpsicológico a lo intrapsicológico-, de la dependencia del sujeto a la independencia, de la regulación externa a la autorregulación. Supone, en última instancia, su desarrollo cultural, es decir, recorrer un camino de progresivo dominio e interiorización de los productos de la cultura (cristalizados en los conocimientos, en los modos de pensar, sentir y actuar, así como de los modos de aprender) $\mathrm{y}$ de los instrumentos psicológicos que garantizan al individuo una creciente capacidad de control y transformación sobre su medio y sobre sí mismo.

- El proceso de aprendizaje posee tanto un carácter intelectual como emocional. Implica a la personalidad como un todo. En él se construyen los conocimientos, destrezas, capacidades, se desarrolla la inteligencia, pero de manera inseparable, este proceso es la fuente del enriquecimiento afectivo, en este se forman los sentimientos, valores, convicciones, ideales, emerge la propia persona y sus orientaciones ante la vida.

- Aunque el centro y principal instrumento del aprender es el propio sujeto que aprende, aprender es un proceso de participación, de colaboración y de interacción. En el grupo, en la comunicación con los otros, las personas desarrollan el autoconocimiento, el compromiso y la responsabilidad, individual y social, elevan su capacidad para reflexionar divergente y creadoramente, para la evaluación crítica y autocrítica, para solucionar problemas y tomar decisiones. El papel protagónico y activo de la persona no niega, en resumen, la mediación social (Castellanos 25).

Como se puede apreciar en la cita, una de las ideas medulares de la concepción del aprendizaje desarrollador es que se aprende estableciendo relaciones significativas, tema en el que se centra el presente trabajo.

\section{Aprendizaje significativo: una concepción a favor de la calidad de la enseñanza}

Ausubel, Novak y Hanesian desarrollaron la concepción del aprendizaje significativo, la cual expresa una antigua idea pedagógica. Para ellos (Ausubel, Novak y Hanesian 3) solo perdura y es verdaderamente útil el aprendizaje que es significativo, es decir, el que provoca que el nuevo contenido adquiera para el estudiante un determinado sentido, en sentido general y amplio, a partir de los conocimientos, actitudes, motivaciones, intereses y su experiencia previa. Para ello es imprescindible el establecimiento de relaciones entre aprendizajes, entre los nuevos contenidos y el mundo afectivo y motivacional de los estudiantes, entre los conceptos ya adquiridos y los nuevos que se forman, entre el conocimiento y la vida, entre la teoría y la práctica. Esta relación significativa es el punto de partida desde el cual el contenido de los nuevos aprendizajes toma un verdadero valor para la persona, y aumenta las posibilidades de que dicho aprendizaje sea duradero, recuperable, generalizable y transferible a nuevas situaciones (características esenciales de un aprendizaje eficiente), así como de que pase a formar parte del sistema de convicciones del sujeto. Si el aprendizaje es significativo, se convierte en una fuerza motivacional que lo estimula.

Elaprendizaje significativo presupone(Ausubel, Novak y Hanesian 15) que el alumno manifiesta una disposición para relacionar sustancialmente, es decir, aquello que incorpora a la estructura cognitiva es la sustancia del nuevo conocimiento, de las nuevas ideas, no las palabras precisas usadas para expresarlas. El mismo concepto o la misma proposición pueden expresarse de diferentes maneras a través de distintos signos o grupos de signos, equivalentes en términos de significados. Asimismo, un aprendizaje significativo no puede depender del uso exclusivo de determinados signos en particular. El conocimiento previo sirve de matriz "ideacional" y organizativa para la incorporación, comprensión y fijación de nuevos conocimientos cuando estos "se anclan" en conocimientos específicamente relevantes, preexistentes en la estructura cognitiva. Pueden aprenderse significativamente y retenerse nuevas ideas, conceptos o proposiciones, en la medida en que otras ideas, conceptos o proposiciones - específicamente relevantes e inclusivos-, estén adecuadamente claros y disponibles en la estructura cognitiva del sujeto y funcionen como puntos de "anclaje" a los primeros. El nuevo material es potencialmente significativo para el aprendizaje con su estructura cognoscitiva, es decir, relacionable con la estructura de conocimiento sobre una base no arbitraria y no al pie de la letra.

Así, pues, si la intención del alumno consiste en memorizar arbitraria y literalmente (esto es, como una serie de palabras relacionadas de forma caprichosa), 
independientemente de la significatividad potencial inherente a la proposición particular, tanto el proceso de aprendizaje como sus resultados serán carentes de significado y mecánicos. A la inversa, si la tarea no es potencialmente significativa, y no es relacionable con la estructura cognoscitiva del alumno, aunque su actitud sea significativa el resultado del aprendizaje posiblemente no será significativo.

La memorización mecánica de definiciones de conceptos o proposiciones, sin el reconocimiento del significado de las palabras de la definición, ilustra lo expuesto. Un estudiante podría aprender la Segunda Ley de Newton, según la cual la sumatoria de las fuerzas que actúan sobre un cuerpo es directamente proporcional a la masa y a la aceleración. Sin embargo, esta proposición no será significativamente aprendida a menos que el estudiante ya sepa los significados de los conceptos de sumatoria, fuerza, directamente proporcional, masa o aceleración, y a menos que trate de relacionar estos significados con lo que estipula la Segunda Ley de Newton.

Infelizmente, los alumnos han constatado que algunos profesores admiten como correctas las respuestas que son repetidas literalmente, lo que conduce a la tendencia al aprendizaje mecánico y memorístico. Otras razones pueden ser el miedo al fracaso, la poca confianza en su capacidad para aprender significativamente o comprender determinado contenido.

La significatividad es lógica y es psicológica. La primera se refiere a la relación coherente entre los conocimientos y depende únicamente del material. La significatividad psicológica comprende la relación de los conocimientos con las necesidades de los sujetos y con su contexto. De ella depende que los contenidos se hagan significativos.

\section{Indicadores de aprendizaje significativo}

A continuación se presentan los indicadores de aprendizaje significativo a partir de la teoría de David Ausubel sobre el tema. Incluyen elementos de orden práctico a partir del efecto de la calidad del aprendizaje en los estudiantes. Contemplan la idea de la pedagogía y la didáctica cubanas acerca de cómo la enseñanza debe influir en toda la personalidad y se hace para preparar para la vida, en un contexto social concreto. Son indicadores de resultado, definidos por la autora para la investigación que dio origen al artículo en particular. Se emplearon en la búsqueda de información relevante de la etapa de diagnóstico, y con el fin de definir acciones concretas de la propuesta didáctica, pues en el proceso de enseñanza-aprendizaje se proponen transformaciones en esos indicadores con el propósito de lograr la significatividad del aprendizaje.

\section{Indicadores}

- Relación del contenido con situaciones y fenómenos de la realidad.

- Reconocimiento y fundamentación de la utilidad de lo aprendido.

- Comprensión y explicación del nuevo contenido a través de su relación con los saberes anteriores.

- Utilización del conocimiento para explicar o resolver problemas vinculados a las actividades más significativas que realiza el sujeto.

- Actitud positiva hacia el aprendizaje de los contenidos.

- Interés en aprender.

- Incidencia integral en la personalidad del sujeto.

\section{Metodología}

En la investigación se emplearon diferentes métodos teóricos y empíricos. Entre los primeros, se empleó el método histórico-lógico a fin de sistematizar las concepciones teóricas que existen acerca del aprendizaje significativo en general, y de la Física en particular, y el análisis y la síntesis de manera que sea posible integrar la información de la bibliografía consultada y los datos recopilados con las técnicas empíricas. El análisis de documentos permitió el examen de los programas y orientaciones metodológicas de la asignatura "Física $10 .^{\circ}$ grado", con el fin de precisar los objetivos y los contenidos que se imparten y las sugerencias que se ofrecen para su enseñanza. Se estudiaron además documentos que ofrecen información acerca de la aplicación de elementos de la Física en la preparación de los atletas y el entrenamiento de diferentes deportes.

El método sistémico-estructural fue utilizado con el fin de elaborar un sistema de recomendaciones metodológicas a partir de los objetivos de las unidades del programa.

\section{Resultados}

\section{Análisis de los resultados de la investigación empírica}

Fueron entrevistados dos profesores de Física y cinco entrenadores de la Escuela de Formación de Atletas 
de Alto Rendimiento (esfaAr) "Cerro Pelado", así como fueron encuestados 28 estudiantes que culminaron el grado décimo: de estos 38 habían recibido el contenido con la profesora y 14 lo cursan actualmente.

Los objetivos de la encuesta fueron conocer:

- La importancia que tiene la Física para los estudiantes.

- Qué contenidos consideran importantes.

- Qué más les gustaría aprender relacionado con los contenidos de la asignatura.

- Si la asignatura los ayuda a comprender fenómenos o situaciones naturales y de la vida en general.

- La utilidad que perciben de la asignatura.

- Si han podido relacionar lo aprendido en Física con su práctica deportiva. Deben especificar qué contenidos.

El 50\% de los estudiantes encuestados reconoce que todos los contenidos de la asignatura son importantes y que les gustaría profundizar más en ellos. Del resto, un $20 \%$ encuentra algunos contenidos importantes para ellos y otros no.

Los contenidos relacionados con el desplazamiento, la velocidad, la aceleración y la fuerza están entre los contenidos más importantes, según los encuestados.

Aunque el 85\% de la muestra reconoce la utilidad de la Física, no aprecian su importancia para el deporte que practican y no saben relacionar los contenidos que reciben en clases con los fenómenos o aspectos específicos de este. A pesar de que recibieron la materia en la ESFAAR "Cerro Pelado", y algunos contenidos fueron relacionados con aspectos de la práctica deportiva, es deficiente la significatividad de la Física para estos alumnos.

Se hicieron, además, entrevistas grupales con los estudiantes, y el principal resultado es que hasta ese momento como tendencia no referían identificar el sentido de la asignatura (significatividad).

\section{Análisis de las entrevistas a los docentes}

Al entrevistar a los profesores se indagó acerca de:

- Los contenidos más comprendidos por los alumnos de décimo grado.

- Los contenidos más aceptados por los alumnos de décimo grado.

- Los contenidos más difíciles de comprender y de relacionar con la vida por los alumnos.
- La relación de los contenidos con la actividad deportiva, la vía utilizada para identificarla y el tratamiento metodológico utilizado.

- Los contenidos que los alumnos han podido relacionar con la actividad deportiva.

- Las propuestas para mejorar la asignatura, el programa o su aplicación.

Los contenidos de décimo grado más comprendidos por los estudiantes, según los profesores, son: el análisis cinemático de los movimientos, el movimiento rectilíneo uniforme y el movimiento rectilíneo uniformemente variado. También el análisis mecánico, las leyes de Newton, la fuerza magnética de Lorentz, la formulación matemática y la aplicación de la regla de la mano derecha; los contenidos referidos a trabajo y energía, así como las leyes de conservación de la energía mecánica.

Entre los contenidos más difíciles dentro de la cinemática está el movimiento curvilíneo (proyectiles). También les resulta complejo entender la ley de conservación de la cantidad de movimiento y la ley de gravitación universal.

El movimiento curvilíneo se reconoce como de gran utilidad y aplicación en deportes como el baloncesto, el lanzamiento de la jabalina, del disco, de la bala y del martillo, para los que es necesario saber el alcance y la efectividad de los lanzamientos. Presentan gran dificultad para comprender y aplicar en este contenido. No advierten que el movimiento curvilíneo no es más que un movimiento en que intervienen el movimiento rectilíneo uniforme y el movimiento rectilíneo uniformemente variado.

En atletismo son necesarios temas como velocidad, rapidez, desplazamiento; en gimnasia rítmica, lo es el movimiento circular al realizar los giros.

Los profesores subrayan que no es preciso cambiar el programa, pero que se debe hacer más énfasis en la aplicación de la Física a los deportes y buscar un mayor vínculo en las clases que el contenido lo permita. Sugieren que se supriman algunas demostraciones matemáticas complejas que desestimulan el interés por la Física. Añaden como la carencia de laboratorios afecta la realización de demostraciones necesarias para una adecuada calidad de la clase, pero tampoco se aprovechan las posibilidades que ofrecen los deportes para apoyar la demostración de los contenidos.

Indican que, como parte de la preparación metodológica, el profesor debe intercambiar con los 
especialistas en deportes a fin de establecer los vínculos posibles.

\section{Análisis de las entrevistas a los entrenadores}

Fueron entrevistados entrenadores de sable, judo, gimnasia y pesas. Se indagó acerca de:

- Los conocimientos de Física que aplican en los entrenamientos.

- Los criterios de los estudiantes sobre la asignatura "Física".

- Las sugerencias para un mejor empleo de los conocimientos la Física en el entrenamiento deportivo.

Los entrenadores de sable destacan que los contenidos físicos son imprescindibles, tanto para las acciones defensivas como para las ofensivas. Temáticas tales como desplazamiento, fuerza, aceleración, empuje, movimiento curvilíneo, velocidad de traslación, velocidad máxima, inercia en la caída, equilibrio, acción y reacción hallan su aplicación en este deporte y en las explicaciones de las ejecuciones. Todos los entrenadores valoran la importancia de la asignatura para su labor, pero manifiestan que si bien los estudiantes-atletas aprecian el valor que tienen los contenidos de Física en el momento del entrenamiento, no así cuando reciben la clase. Proponen un mayor vínculo entre ellos y los profesores de Física.

El entrenador de judo manifiesta que entre los contenidos de Física que se vinculan más estrechamente a su deporte están los conceptos de centro de gravedad, palanca, ángulos para la ejecución de las técnicas, movimiento rotacional, fuerza en los planos horizontal y vertical, velocidad, empuje, etc. Los entrenadores recomiendan que se les indiquen tareas a los estudiantes-atletas, de manera que apliquen los contenidos de Física a sus deportes.

En general, aunque los entrenadores aplican los conocimientos de Física, los estudiantes-atletas son incapaces de relacionarlos con su práctica deportiva.

\section{Análisis del programa de Física de décimo grado}

En un análisis del programa de Física se pudo determinar qué contenidos tienen una mayor presencia en la práctica de determinados deportes.

De manera general se concluye lo siguiente:

- A través del diagnóstico, se pudo constatar que la Física resulta poco significativa para una buena parte de los estudiantes-atletas que cursaron el décimo grado en la ESFAAR "Cerro Pelado".

- No se ve una intención de hacer significativos los contenidos de Física en las clases a través de su vinculación con la práctica deportiva. Esta limitación tiene su origen en la inexistencia de una estrategia didáctica para este fin.

- El escaso intercambio entre los profesores de Física y los entrenadores deportivos ha imposibilitado una mayor explotación de los aspectos del deporte relacionados con la Física, a fin de lograr una mayor significatividad de la asignatura para los alumnos-atletas.

\section{Conclusiones}

\section{Hacia un aprendizaje significativo de la Física}

La calidad del proceso de enseñanza-aprendizaje de la Física no escapa a la problemática general de la calidad de la educación en Cuba. Al examinarlo sobre la base de la teoría del aprendizaje significativo de Ausubel, particularmente lo que se refiere a la contraposición entre el aprendizaje significativo y el aprendizaje mecánico, se advierte como el alumno no logra relacionar de forma esencial y no arbitraria lo que trata de aprender con lo que ya conoce, y no puede utilizar el conocimiento de forma novedosa o innovadora. Estas dificultades subsisten en los estudios posteriores, se arrastran en los diversos niveles de enseñanza y dificultan el aprendizaje de la asignatura. El aprendizaje mecánico, es decir, de información almacenada arbitrariamente, sin interactuar con conocimientos preexistentes (el simple aprendizaje memorístico de fórmulas en Física, por ejemplo), invade muchas veces la enseñanza-aprendizaje de la asignatura.

Al pasar el estudiante de un nivel educacional a otro sin los conocimientos indispensables que le permitan vencer etapas posteriores, se incrementa constantemente el porcentaje de conocimientos adquiridos en la forma mecánica descrita. Como consecuencia, tal situación generalmente conduce a la incapacidad generalizada de cualquier estudiante para adquirir nuevos conocimientos significativos $y$, finalmente, al fracaso académico.

Las ventajas del aprendizaje significativo para la enseñanza de la Física son, a juicio de la autora, las siguientes: 
- Este tipo de aprendizaje modifica la estructura cognitiva del alumno mediante su reacomodación, de manera que este sea capaz de integrar la nueva información; por tanto, el alumno tiene una retención más duradera de los conceptos físicos.

- La adquisición de nuevos conocimientos de Física se produce con mayor facilidad relacionando los ya aprendidos con los nuevos en forma significativa, ya que al estar claramente presentes en la estructura cognitiva se facilita su relación con los nuevos contenidos.

- Al ser de interés para el alumno la nueva información sobre los conceptos físicos, esta se conserva y no se olvida fácilmente.

- Es un aprendizaje activo, pues se construye con base en las acciones y las actividades de aprendizaje de los propios alumnos.

- Es personal, pues la significación de los aprendizajes depende de los recursos cognitivos del alumno, de sus necesidades, de su interés y de su realidad.

La significatividad de la asignatura se eleva para los estudiantes-atletas en la medida en que:

- Se aprecia la relación entre los contenidos con situaciones y fenómenos de la realidad, partiendo de la identificación del vínculo con su principal actividad: la deportiva.

- Se logra una actitud positiva hacia el aprendizaje de los contenidos e interés por aprender en la mayoría de los estudiantes, lo cual incide en la integralidad de la personalidad del sujeto.

En el plano de lo metodológico:

- Se alcanza la comprensión y explicación del nuevo contenido a través de su relación con los saberes anteriores.

- Se emplea en cada unidad el contenido para explicar o resolver problemas vinculados a la actividad más significativa que realizan estos alumnos-atletas.

El planteamiento que se hace en la propuesta no va en dirección de hacer significativo directamente un concepto, sino su relación funcional con la práctica del deporte. Es decir, es esencial que se relacionen los contenidos y las leyes de la Física con situaciones deportivas concretas y se demuestre cómo la aplicación correcta en estas regularidades contribuye (y a veces de manera decisiva) al éxito deportivo. Esto debe dominarlo el alumno ante el entrenador. Por eso es importante que el estudiante, cuando llegue a la carrera de Licenciatura en Cultura Física, tenga las bases suficientes en la asignatura de Física, a fin de comprender su aplicación en los entrenamientos y en las clases de Educación Física.

\section{Referencias}

Ausubel, David, Joseph Novak y Helen Hanesian. Psicología educativa: un punto de vista cognoscitivo. México: Editorial Trillas, 1993. Impreso.

Castellanos, Doris. La comprensión de los procesos del aprendizaje: apuntes para un marco conceptual. La Habana: Centro de Estudios Educacionales ISPEJv, 1999. Impreso.

Chávez Rodríguez, Alberto Justo y Gustavo Deler Ferrera. Antología del pensamiento educacional de la revolución cubana. Web. Junio de 2015.

"Fundamentos de la investigación educativa." Maestría en Ciencias de la Educación. Módulo I. Segunda parte. Editorial Pueblo y Educación. Impreso.

Instituto Central de Ciencias Pedagógicas. Propuesta sintetizada del modelo actuante de la escuela secundaria básica. Impreso.

González Fernández, Ana María. La investigación educativa desde un enfoque dialéctico. La Habana: Iplac, 2005. Web. Junio 14 de 2015.

Ministerio de Educación. Programa de Física Décimo Grado Vigente desde 2005-2006. Web.

Pérez Rodríguez, Félix. La Escuela Deportiva Cubana: Un enfoque de procesos. Web. Junio 14 de 2015.

Ministerio de Educación. Programas Décimo grado Educación preuniversitaria: primer año Educación Técnica y Profesional. La Habana: Editorial Pueblo y Educación, 2006.

Unesco. Declaración de México sobre las politicas culturales Conferencia mundial sobre las políticas culturales. Conferencia, 26 de julio -6 de agosto de 1982. Web. Junio de 2015 . 